\title{
Sur les milieux poreux et les coefficients de porosité
}

\section{Porous media and coefficients of porosity}

\author{
I'AR MA Min-YUAN \\ INGÉNILUR A LA SOGILEKAH
}

\begin{abstract}
La porosité et les coefficients de porosité sont un sujet fort particulier dans l'hydraulique, mais très complexe. Il a suscité d'intéressantes études et des controverses passionnées. et il provoque aujourd'hui mếme des discussions, surtout en ce qui concerne les valeurs à ntiliser vour les divers coefficients.

C'est à propos de ceux-ci que l'auteur résume ici ses diverses réflexions sur le sujet. Et dans son exposé, il montre implicitement comment on pent mienx saisir le sens de «porosité 》et choisir ensuite les valeurs des coefficients d'une. façon plus adéquate.
\end{abstract}

On sait que les phénomènes d'écoulement dans les milieux poreux ont leur influence sur la solidité des ouvrages des installations hydroélectriques. Et pour étudier ces influences, nous avons besoin des coefficients de porosité pour faire des calculs. C'est ainsi que nous avons été amené à consulter les ouvrages de MM. Tison [1], Terzaghi [2], Serafim [3]... ainsi que des études de Graton et Fraser ]4], Harza [5], Leliavsky [6]. Nous exposons ci-après certaines de nos réflexions provoquées par ces lectures.

$$
\text { ******* }
$$

Dans certains exposés, on attache pour raison de commodité le sens de «vide» géométrique au sens de porosité. C'est ainsi que généralement on définit, pour un milieu considéré, le rapport géométrique :

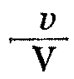

\begin{abstract}
Porosity and coefficients of porosity are a very particular and complex aspect of hydraulics, which has given rise to interesting studies and much controversy. Opinions are still divided on some points, especially regarding the values of the various coefficients involved.

The anthor states his own opinion on this last point and shows in his exposé how to gain a better understanding of the meaning of porosity and thas give the coefficients more representalive values.
\end{abstract}

ò̀ $v=$ le volume du vide géométrique contenu dans le milieu considéré,

$\mathrm{V}=$ le volume géométrique du milieu considéré.

On appelle ce rapport: porosite, ou coeffcient volumique de porosité, du milieu considéré; on le désigne souvent par $m$. Cette facon de définir la porosité est simple et commode, mais elle manque de réalisme. Et nous en indiquerons plus loin la raison.

Ainsi, pour un milieu poreux formé par des grains de forme sphérique et sensiblement égaux entre eux, on peut, dans des études, assimiler ce milieu à un amas de sphères pleines de rayon $R$. Une fois cette idéalisation adoptée, il est naturel de voir si l'on peut calculer le coefficient $m$ d'après la définition donnée ci-dessus.

Pour faire un calcul, il faut donner une hypothèse sur l'entassement de l'amas. En effet, en admettant que les sphères soient pleines et en con- 
tact entre elles de telle façon que les droiles passant par le centre et les points de contact forment un système d'axes triorthogonaux (voir les figures 1 et 2), on sait que, physiquement, cet

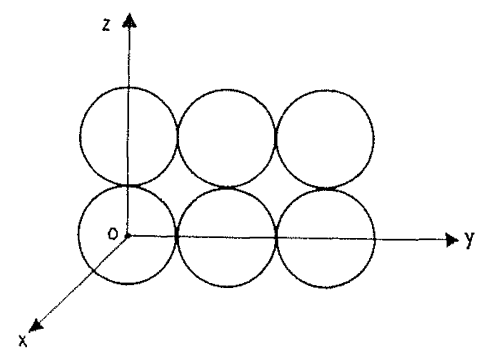

Fig. 1

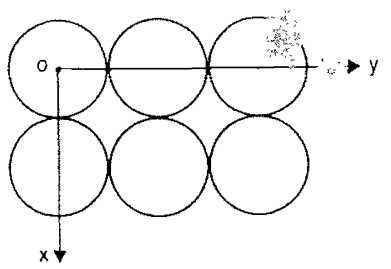

FIG. 2

entassement est en équilibre, mais instable. Or, des caleuls faits montrent que dans ce cas :

$$
m=1-\frac{\pi}{3 \times 2}=0,476
$$

De l'entassement défini par les figures 1 et 2 , si on garde le dispositif dans le plan xoy, mais si, en revanche, on déplace les sphères (dont les centres sont de $z=2 \mathrm{R}$ ), dans la direction oy, de la distance $\mathrm{R}$, pour avoir dans le plan yoz l'arrangement figuré dans la figure 3 , il est à re-

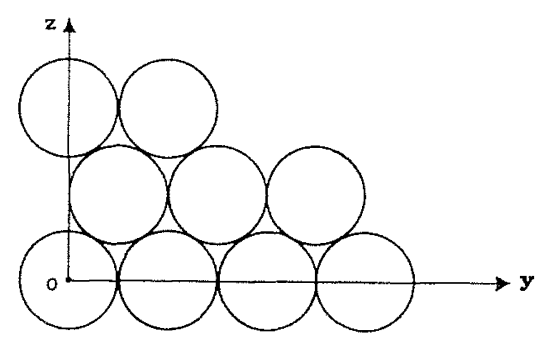

FIG. 3

marquer que cet arrangement est en équilibre, instable lui aussi. Mais dans ce cas, on a :

$$
m=1-\frac{\pi}{3 \sqrt{3}}=0,396
$$

Si, à partir de l'entassement défini par les figures 2 et 3 , on déplace les sphères dont le centre est de $z \sqrt{3 \mathrm{R}}$ dans la direction $o x$, telle que l'aspect de celle couche de sphères dans le plan xoy est figurée dans la figure 4, on trouve alors un entassement stable, et dans ce cas :

$$
m=1-\frac{\pi}{3 \sqrt{2}}=0,259
$$

Nous signalons en passant que ce dernier entassement est le même que l'entassement des

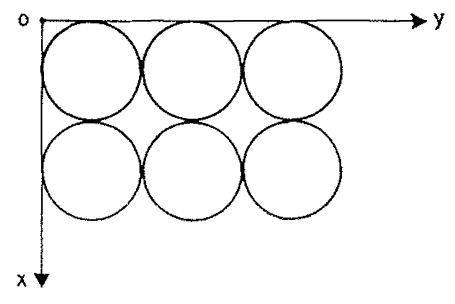

Fig, 4

sphères en pyramide à base de triangle régulier. On peut montrer ceci par le calcul de $m$, ou directement par des considérations géométriques.

Les valeurs théoriques obtenues ci-dessus sont bien connues et sans reproches, mais malgré ces calculs sans reproches, nous nous sommes demandé quelle est la validité de ces considérations géométriques.

$$
* * *
$$

En réalité, l'entassement naturel d'un milieu poreux homogène formé par des grains sphériques assimilables à un amas de sphères pleines admet rarement 0,259 coinme coefficient de porosité volumique, puisque, dans le milieu naturel formé par des sphères parfaites, les sphères ne sont pas toutes entassées d'une façon serrée jusqu'à l'idéal; et l'entassement naturel peut être considéré comme un mélange de parties à coefficients de porosité volumique différents et qui sont de :

\subsection{6 \\ 0,396 \\ 0,259}

respectivement. De plus, on peut même admettre que, dans la nature, la possibilité de trouver des parties à $m=0.476$ est très faible, en sorte que, pratiquement, on peut prétendre que l'entassement naturel est formé par deux catégories de parties :

- « catégorie $A$ » à coefficient de porosité volumique $=0,396$,

- 《 catégorie B », à coefficient de porosité volumique $=0,259$. 
C'est ainsi que, si la « catégorie A 》 représente $50 \%$ du volume total, la porosité volumique est de 0,327 et si la 《 catégorie $\mathrm{A}$ » est de $25 \%$, alors la porosité volumique est de 0,293 .

On voit alors que l'entassement naturel s'approche plus de l'état idéal lorsque son coefficient volumique de porosité s'approche de 0,259. Mais il est à remarquer qu'avec des sphères parfaites, on arrive très difficilement à un tel entassement, à cause des frottements non nuls entre les sphères, ce qui revient à dire que, même avec des sphères pleines de rayons absolument identiques, de dimensions ordinaires, on n'arrive qu'à fairc un milieu poreux, théoriquement homogène, mais d'un $m$ plus grand que 0,259 et assez petit par rapport à 0,396 , car pour $m=0,396$, on a un état homogène très instable vis-à-vis des secousses.

A notre point de vue, sans expériences failes, on peut raisonnablement concevoir des milieux poreux homogènes stables dans la nature, de coefficient de porosité volumique de l'ordre de 0,28 à 0,3 , mais nous disons tout de suite que la connaissance de cette limite est très intéressante, et nous en indiquerons plus loin la raison.

$$
\text { * }
$$

Jusqu'ici nous avons considéré la question de porosité du point de vue de la géométrie.

Or, physiquement, les matières sont considérées comme formées de molécules de formes plus ou moins sphériques. Et, pour le présent exposé, on peut considérer que les milieux matériels sont formés par des molécules en contact entre eux, et que les molécules d'eau peuvent être assimilées à des sphères d'un diamètre de l'ordre de $3,5 \cdot 10^{-8} \mathrm{~cm}$; de même, les molécules de fer ou de silice sont assimilables à des sphères de diamètres de l'ordre de 2,5 à $3 \cdot 10^{-8} \mathrm{~cm}$. On voit alors qu'il est possible de considérer les aciers, les silices, comme un milieu plein de vide, c'està-dire poreux; mais il est inconcevable de dire que des molécules d'eau chimiquement libres peuvent s'emmagasiner dans ce genre de milieu. Et dans ce cas, le coefficient volumique de porosité doit être nul, c'est-à-dire :

$$
m=0
$$

ce qui montre que, dans l'hydraulique, si nous considérons des milieux poreux formés par des grains sphériques de diamètre $d$ entassés d'une façon idéale, alors on voit que $m$ doit varier de 0 jusqu'à 0,259 en suivant l'augmentation de l'échelle de $d$. Dans la nature, il n'est pas sans raisons d'admettre que pour $d$, de l'ordre de de $10^{-3} \mathrm{~cm}$ et même de l'ordre de $10^{-4} \mathrm{~cm}$ ou $10^{-5} \mathrm{~cm}, m$ peul atteindre la valeur théorique : 0,259. Ainsi, pour des milieux poreux formés par des grains de dimensions ordinaires, de forme sphérique, assimilables à des sphères, il est tout à fait réaliste de penser avoir dans l'hydraulique :

$$
m=0,259
$$

ou plus grand, comme nous l'avons expliqué plus haut.

$$
*
$$

Avant de poursuivre les examens d'autres aspects de la porosité, nous signalons ici le point suivant, relatif à la mesure de $m$ dans les laboratoires.

Dans la pratique, pour la mesure du vide laissé par des sphères de rayon $R$ entassées dans le milieu, on entasse souvent les sphères dans une cuve cubique, dont les côtés sont beaucoup plus grands que $R$, et on mesure la quantitć d'eau que peut contenir encore la cuve par des méthodes appromrées. Nous savons bien que ces mesures ne se font pas sans peine, mais il est à remarquer que le $m$ ainsi trouvé n'est pas exact si l'on a pris seulement la précaution que le côté du cube soit beaucoup plus grand que R. En effet, il est très juste de penser que, dans la cuve, au contact des parois et à leur approche, les sphères sont moins bien entassées que celles qui se situent plus à l'intérieur du cube. Si l'on considère que cet état de choses correspond à un volume $v$ (de coefficient de porosité $M$ par surface de paroi) où les sphères s'entassent plus mal que celles situées plus à l'intéricur du cube et dont le coefficient de porosité est $m$ (on sait que $M>m$ ), on voit que (le volume géométrique du cube étant $V$ et le coefficient de porosité de la cuve entière étant $m_{1}$ ), on a :

$$
6 v \cdot \mathrm{M}+(\mathrm{V}-6 v) . m=\mathrm{V} m_{1}
$$

ce qui montre bien que $m_{1}$ n'est pas la valeur $m$ que l'on veut obtenir.

A notre point de vue, il est très facile de trouver au moins thériquement la vraie valeur de $m$. En effet, on peut faire une deuxième mesure en prenant une autre cuve à base du même carró que la première cuve, mais de hauteur double. Si la deuxième mesure donne $m_{\text {. }}$ conmme coefficient de porosité volumique, on sait alors que $m_{2}$ s'approche plus près de $m$ que $m_{1}$. Or, dans ce cas, on a :

$$
10 v \mathrm{M}+(2 \mathrm{~V}-10 v) m=2 \mathrm{~V} m_{2}
$$

On déduit de ces deux mesures que :

$$
2 v \mathrm{M}-2 \mathrm{vm}=2 \mathrm{Vm}_{1}-2 \mathrm{Vm}_{2}
$$

et :

$$
m \cdot \mathrm{V}=\mathrm{V} \cdot m_{1}-3\left(2 \mathrm{~V} \cdot m_{1}-2 \mathrm{~V} \cdot m_{2}\right)
$$

Et on voit que la vraie valeur du coefficient de 
porosité volumique à mesurer est donnée alors par la relation très simple :

$$
m=6 m_{2}-5 m_{1}
$$

Prenons par exemple :

$1^{\circ} m_{1}=0,50 ; m_{2}=0,48$,

on $a: m=2,88-2,5=0,38$;

$2^{\circ} m_{1}=0,33 ; m_{2}=0,32$,

$$
\text { on a : } m=1,92-1,65=0,27 \text {. }
$$

ces exemples numériques montrent déjà qu'il y a des précautions à prendre dans les mesures faites dans les laboratoires d'essais pour avoir des résultats plus conformes à la réalité.

D'ailleurs, la formule $m=6 m_{2}-5 m_{1}$ montre encore que si l'erreur maximale commise dans les mesures de $m_{2}, m_{1}$ est de 0,01 , l'erreur maximale commise sur la valeur de $m$ peut alors atteindre :

$$
(6+5) 0,01=0,11
$$

ceci illustre parfaitement le fait que la détermination pratique de $m$ dans la nature est une opération très délicate, même dans le cas le plus simple.

$$
* *
$$

Nous avons examiné les divers aspects du coefficient de porosité volumique d'un milieu poreux idéal, formé par des sphères pleines de même rayon $\mathrm{R}$. Et nous allons maintenant examiner pour ce même milieu la question du coefficient superficiel de porosité.

En partant de nouveau de la notion du vide géométrique pour définir le cocfficient superficiel de porosité, on sait que ce coefficient doit être un rapport de deux surfaces géométriques.

Pour ce faire, on prend un plan qui coupe le milieu poreux formé par des sphères pleines. Alors, on a :

$\mathrm{S}_{i}=$ la surface totale des cercles dans le plan,

$\mathrm{S}_{e}=$ la surface totale des zones intercercles dans le plan; on appelle coefficient superficiel de porosité du milieu considéré, le rapport :

$$
\frac{\mathrm{S}_{e}}{\mathrm{~S}_{i}+\mathrm{S}_{e}}
$$

qu'on désigne quelquefois par $\eta$.

En adoptant cette définition géométrique, nous voyons immédiatement que n ainsi défini, pour le milieu poreux formé par des sphères pleines identiques, dépend : $1^{\circ}$ de la grandeur de la portion considérée $\mathrm{S}$ du plan de repère;

$2^{\circ}$ de la direction et de la position du plan de repère.

En effet, si l'on prend l'entassement idéal des sphères défini par les figures 2 et 4 , on voit qu'en tendant le plan de repère l'infini, on a, suivant l'orientation de ce plan :

$1^{\circ}$ Le plan est parallèle à $x o y$ et de côté $z$ :

$$
n=\left\{\begin{array}{r}
1-(\pi / 4)\left[1-(z / \mathrm{R})^{2}\right] \\
\text { pour } 0 \leqslant z \leqslant(\sqrt{2}-1) \mathrm{R} \\
1-(\pi / 2) \cdot(z / \mathrm{R})(\sqrt{2}-z / \mathrm{R}) \\
\operatorname{pour}(\sqrt{2}-1), \mathrm{R} \leqslant z \leqslant \mathrm{R}
\end{array}\right.
$$

\begin{tabular}{|c|c|c|c|}
\hline$y / \mathrm{R}$ & 0 & 0,5 & 1 \\
\hline$\eta$ & 0,445 & 0,167 & 0,445 \\
\hline
\end{tabular}

c'est-à-dire :

\begin{tabular}{c|cccccc}
$z / \mathrm{R}$ & 0 & 0,2 & $\begin{array}{c}0,414 \\
(\sqrt{2}-1)\end{array}$ & $\begin{array}{c}0,707 \\
(1 / \sqrt{2})\end{array}$ & 1 \\
\hline$r_{i}$ & $0,215 \nearrow 0,246 \nearrow$ & 0,35 & & 0,215 & $\nearrow 0,35$
\end{tabular}

$2^{\circ}$ Le plan est perpendiculaire à oy :

$\eta_{i}=1-\frac{\pi}{4 \sqrt{2}}\left[1+2-\frac{y}{\mathrm{R}}-2\left(-\frac{y}{\mathrm{R}}\right)^{2}\right] ; \quad 0 \leqslant y \leqslant \mathrm{R}$

c'est-à-dire :

Et il est à remarquer, comme nous l'avons dit, que l'entassement considéré ici est le même que celui d'entassement en pyramide à base de triangles réguliers. Mais si l'on considère un plan in-
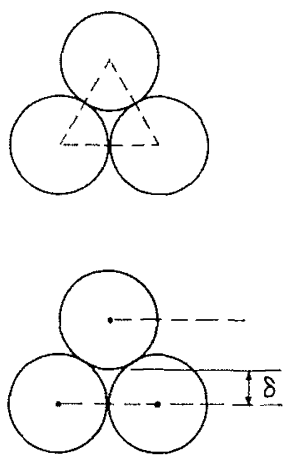

Fic. 5

fini parallèle à celui passant par les centres de trois sphères identiques en contact et de distance $\delta$ par rapport au plan des trois centres indiqués, on a : 


$$
\eta=\left\{\begin{array}{r}
1-\frac{\pi}{2 \sqrt{3}}\left[1-\left(\frac{\delta}{\mathrm{R}}\right)^{2}\right] \\
\text { pour } 0 \leqslant \delta \leqslant\left(2 \cdot \sqrt{\frac{2}{3}}-1\right) \mathrm{R} \\
1-\frac{\pi}{2 \sqrt{3}}\left[-\frac{2}{3}+4 \sqrt{\frac{2}{3}} \frac{\delta}{\mathrm{R}}-2\left(\frac{\delta}{\mathrm{R}}\right)^{2}\right] \\
\operatorname{pour}\left(2 \sqrt{\frac{2}{3}}-1\right) \mathrm{R} \leqslant \delta \leqslant \mathrm{R}
\end{array}\right.
$$

c'esti-à-dire :

\begin{tabular}{|c|c|c|c|c|c|}
\hline$\hat{\delta} / \mathrm{R}$ & 0 & 0,2 & 0,4 & $(2 . \sqrt{2 / 3}-1)$ & $\sqrt{2 / 3}$ \\
\hline
\end{tabular}

Devant les diverses valeurs de $\eta$ indiquées ici pour un cas très simple de milieu poreux assimilable à un milieu homogène, on est obligé de se demander quelle est la valeur de $\eta$ à utiliser dans des études théoriques où l'on veut assimiler le milieu poreux à un milieu homogène (nous disons homogène au sens mathématique) en vue de faire des calculs.

A ce sujet, deux propositions des plus connues ont été faites. Nous allons les examiner cidessous.

$$
* *
$$

La première proposition est de prendre la moyenne de $\eta$ le long d'une direction donnée (on verra plus loin que cette direction n'entre pas en jeu) sur laquelle l'élément de distance est désigné par $d n$; on prend alors, si $\mathrm{S}$ est une surface plane assez grande, perpendiculaire à la direction considérée, le rapport :

$$
\frac{\int S \eta \cdot d n}{\int S d n}
$$

Il est à remarquer que le rapport ainsi défini varie suivant la valeur de départ de $r_{i}$, de $S$, et de l'intervalle d'intégration de $d n$. Pour des S particuliers et des intervalles particuliers de $n$, on a :

$$
\frac{\int \mathrm{S} \eta \cdot d n}{\int \mathrm{S} d n}=\frac{v}{u}=m
$$

et pour un intervalle quelconque entre $n$ et $S$, suffisamment grands, le rapport considéré diffère très peu de $m$ et on voit ici même que cette valeur ne dépend pas de la direction considérée. Ainsi, on voit quelquefois des auteurs recommander de prendre :

$$
\text { coefficient superficiel de porosité }=m
$$

pour le milieu poreux assimilé à un état idéal.

Cette proposition n'est pas, à notre point de vue, parfaite. En effet, la moyenne ainsi obtenue dépend, comme nous l'avons dit, de $S$ et de l'intervalle d'intégration de $d n$. Or, dans les études, on utilise souvent la notion de coefficient superficiel de porosité, pour examiner des questions liées à la pression locale, aux mouvements des particules d'eau, ce qui demande que la valeur à utiliser soit une moyenne par rapport à toutes les directions (et non une moyenne par rapport à la distance dans une direction donnée) et de plus, soit indépendante de la grandeur de $\mathrm{S}$ considérée (et non dépendante de cette grandeur) lorsque $S$ est petite

\section{D'ailleurs, la proposition :} coefficient superficiel de porosité $=m$

nous conduit à l'interprétation suivante de la réalité :

«Le vide dans le milieu poreux idéalisé se comporte comme s'il était formé de tubes continus de surface totale $m$ fois plus petite dans toutes les directions. $\gg$

Or, physiquement, il est très difficile d'imasiner un tel milieu.

La deuxième proposition consiste à prendre : $m^{2 / 3}$ comme coefficient superficiel de porosité.

A notre point de vue, ceci satisfait plus à l'esprit. En effet, pour idéaliser le milieu poreux, il est plus raisonnable de supposer qu'en remplaçant le vide par des molécules d'eau, ces molé. cules se dispersent d'une façon homoggène, dans tout volume partiel quelconque. De la sorte, si dans un volume rempli d'eau (mais dans lequel seront ultérieurement immergés des corps poreux), une surface $S$ coupait $N$ molécules d'eau, cette même surface ne rencontrera, dans le milieu poreux dont le vide est occupé par l'eau, que $m^{2 / 3} \mathrm{~N}$ molécules d'eau, en admettant que l'eau se disperse dans ce milieu d'une facon homogène.

Donc le coefficient superficiel de porosité est alors $m^{2 / 3}$ quelle que soit la surface $\mathrm{S}$ (mais beaucoup plus grande que les échelles moléculaires qui sont de l'ordre de $3 \cdot 5 \cdot 10^{-8} \mathrm{~cm}$ ).

Et on en déduit que pour un milieu poreux homogène, la relation :

$$
\eta=m^{2 / 3}
$$

est théoriquement admissible. C'est ainsi que, pour le milieu poreux idéal des sphères pleines et identiques, de dimension ordinaire, on peut prendre :

$$
\eta=(0,259)^{2 / 3}=0,406
$$

Comparons cette valeur théorique avec les diverses valeurs calculées plus haut; on voit qu'elle est parfaitement acceptable, tout en no- 
tant que dans certaines directions, le $\eta$ réel peut ctre plus grand.

Nous avons considéré jusqu'ici les milieux poreux formés par des grains de forme sphérique, assimilables à des sphères identiques, tout en supposant que les sphères sont pleines (c'està-dire les molécules d'eau n’arrivent pas à pénétrer à l'intérieur des sphères). Nous proposons d'appeler ce genre de porosité «porosité simple».

Et pour des milieux poreux formés par des grains, qui sont à leur tour poreux, nous dirons alors que ces milieux poreux sont $d e$ «porosité composée ».

Donnons un exemple: un milieu poreux formé par des sphires en acier ou en silice pure, de rayon $1 \mathrm{~mm}$, est de porosité simple, car on sait que les molécules d'eau n'arrivent pas à pénétrer à l'intérieur de ces sphères.

Mais, pour le milieu poreux formé, par exemple, par des sphères de rayon $1 \mathrm{~mm}$, ces sphères, dites sphères-grains, son'i composées à leur tour par des petites particules sphériques de diamètres assez petits, par exemple $5.10^{-4} \mathrm{~cm}$. Il est alors raisonnable d'admettre que les molécules d'eau peuvent entrer librement à l'intérieur des sphères-grains et remplissent les vides interparticules. Ce dernier genre de porosité est physique. ment bien différent du premier. Ce qui nous pousse à parler de «porosité composée » et à y porter une attention particulic̀re.

$$
* *
$$

Nous nous plaçons ici dans le genre de porosité composée indiquée ci-dessus et nous supposons que les particules soient bien entassées pour former les sphères-grains et que les sphires-grains soient bien entassées pour former le milieu poreux. D'après les calculs faits pour les milieux porcux, de porosité simple, mais idéaux, il est facile de voir que, pour le milieu poreux de porosité composcée, on a :

$$
m_{c}=m_{s}+\left(1-m_{s}\right) m_{p}
$$

oi $m_{i}$ : coefficient volumique de porosité composée du milieu poreux considérć;

$m_{s}$ : coefficient volumique de porosité simple en admoltant les sphères grains pleins;

$m_{i}$ : coefficient volumique de porosité des particules dans les sphères-grains.

Bien entendu, si $m_{p}=0$, on trouve que $m_{t}=m_{s}$, ce qui montre que $m_{r}$ peut se réduire au coefficient volumique de porosilé simple.

Pour la détermination de $m_{c}$ dans les laboratoires, on doit employer la méthode indiquée plus haut, c'est-à-dire : si $\left(m_{c}\right)_{1}$ donne la mesure de $m_{c}$ pour les échantillons placés dans une cuve cubique,

et si $\left(m_{c}\right)_{2}$ donne la mesure de $m_{c}$ pour les échantillons placés dans une cuve de même base, mais de hauteur double,

la vraie valeur de $m_{c}$ est alors définie par:

$$
m_{c}=6\left(m_{c}\right)_{2}-5\left(m_{c}\right)_{1}
$$

Il est à remarquer que la valeur $m_{c}$ est très variable. En effet, pour les milieux poreux formés de sphères-grains entassées d'une façon parfaite, on a théoriquement :

$$
n \iota_{s}=0,259
$$

et d'autre part, si les particules sphériques (de diamètres assez grands) sont aussi entassées d'une façon parfaite pour former les sphèresgrains, on a aussi :

$$
m_{p}=0,259
$$

On trouve alors que :

$$
m_{c}=0,259+(1-0,259) \times 0,259=0,451
$$

Nous avons eu l'occasion de parler de la limite de $m_{s}$ au début de notre exposé. Or, pour distinguer la porosité simple et la porosité composée, la connaissance de cette limite physique est devenue une nécessité. Il est entendu que l'on peut faire des essais avec des sphères en acier ou en silice de dimensions ordinaires, et que ces essais peuvent donner la limite $\mathrm{M}_{3}$ qui est plus grande que 0,259 .

Ainsi, pour des milieux poreux réels dont les grains sont des sphères identiques, on voit que, suivant la valeur de $m$ obtenue d'après des mesures, il est permis de dire que le milieu considéré est :

$1^{\circ}$ de porosité simple, si $m \leqslant M_{s}$;

$2^{\circ}$ de porosité composée, si $m \geqslant M_{s}$;

3" de porosité simple, mais mal tassée, si $m$ est légêrement supérieur à $\mathrm{M}_{s}$.

Dans la pratique, il est pensable que $m_{s}$ peut atleindre 0,28 à 0,3 . Quant à $m_{p}$, il peut, comme nous l'avons vu, être variable dans l'intervalle 0 et 0,3 , ou même plus grand, suivant la construclion des sphères-grains.

$$
\therefore
$$

Nous allons maintenant examiner des questions relatives au coefficient superficiel de poro- 
sité pour les milicux poreux de «porosité composée 》 indiquée plus haut.

En effet, pour ce genre de milieu poreux assimilable à l'exemple cité plus haut, l'homogénéisation de ce milieu nous incite à prendre dans les études théoriques:

$$
\eta=m_{c}^{2 / 3}
$$

Or, le schéma que nous avons pris pour calculer $m_{c}$ montre un fait physique très important : la situation des molécules d'eau d̀ l'intérieur des sphères-grains n'est pas du tout la même que celle des molécules d'eau emmagasinées entre les sphères-grains. Il est alors raisonnable de penser que la facilité de se déplacer est beaucoup plus grande pour la dernière catégorie de molécules d'eau que pour ceux de la première catigorie. Ce qui revient à dire que, relativement, les molécules d'eau situćes à l'intérieur des sphères-grains sont liées à ces sphères-grains mêmes. Ainsi, pour les études du mouvement permanent des molécules d'eau à travers le milieu poreux, le coefficient superficiel de porosité à utiliser est lié à $m_{s}$, c'est-à-dire que l'on doit faire intervenir :

$$
\eta=m_{s}^{3 / 2}
$$

Mais au point de vue de l'emmagasinage et de la pression, on doit faire intervenir respectivement :

$$
m_{c} \text { et } \eta=m_{c}^{2 / 3}
$$

Par exemple, pour $m_{s}=m_{p}=0,3$, on doit prendre théoriquement :

$$
\begin{gathered}
m_{c}=0.51 \\
\eta=\left(m_{c}\right)^{2 / 3}=0,631
\end{gathered}
$$

Et cette valeur de $\eta$ doit être considérée comme une limite inférieure.

Or, si l'on examine de plus près la poussée hydraulique sur les corps solides qui constituent le milieu poreux, on s'apercoit que si $\mathrm{S}$ est la surface d'une section de référence, et $s$, la sommc, projetée sur $\mathrm{S}$, des surfaces de contact des sphères-grains ou des molécules mêmes $(s$ est très petite par rapport à $S$ ), la pression hydraulique s'applique alors sur :

$$
\mathrm{S}-s^{\prime}
$$

où $s^{\prime}$ est plus grande que $s$, puisque aux alentours immédiats des surfaces de contact $s$, il n'y a pas de molécules d'eau. On a alors :

$$
\eta=1-\frac{s^{\prime}}{\mathrm{S}}
$$

Et la valeur de $\eta$ ainsi obtenue peut atteindre des valeurs proches de 1 .

$$
* *
$$

Nous avons jusqu'ici examiné les cas des milieux poreux formés par des grains sensiblement identiques et de forme sphérique. Le raisonnement peut s'étendre aux cas des milieux poreux formés de grains de formes sensiblement sphériques et de dimensions assez rapprochées.

Quant aux milieux poreux formés par des grains de forme non sphérique, il est très diffcile de fonder des hypotheses simples et d'en chercher des conclusions mathématiques. Mais les considérations exposées ci-dessus pour les milieux poreux formés par des grains-sphères, telles que les précautions pour la mesure de porosité volumique, les sens de porosités simple ou composée ... et les relations entre $\eta$ et $m$, les coefficients à utiliser pour un problème donné... restent valables.

Nous signalons en terminant que l'idée de donner un schéma de grillage aux milieux poreux de grande porosité volumique est très intéressante, mais nous y ajoutons simplement une pelite modification en disant : "aux milieux poreux de grand coefficient volumique de porosité simple, c'est-à-dire de grand $m_{s} »$.

On peut peut-être nous objecter qu'il est difficile de séparer pratiquement $m_{\sigma}$ et $m_{s}$. Mais nous pensons qu'en s'appuyant sur les propriétés différentes, du point de vue hydraulique, des molécules d'eau situées entre grains ou à l'intérieur des grains, on doit arriver pratiquement à distinguer $m_{c}$ et $m_{s}$, avec des valeurs plus ou moins approchées.

\section{BIBLIOGRAPHIE}

11] L. J. Trsos. - « Cours d'hydraulique», Gand, 1953.

[2] K. yon TeRzaGHI. - "Theoretical soil mechanies», Wiley, N.Y., 1943.

[3] J. Laginha Seraris. - «A subpressao nas barragens $\gg$ Lisboa, 1954.

[4] I.. C. Graton et H.J. Fraser. - « Systematic packing of spheres with particular relation to porosity and permeability », The Journal of Geology, V. 43, $\mathrm{n}^{\circ} 8$, part 1 , nov.-déc. 1935 .

[5] L. HARZA. - " The significance of pore pressure in hydraulic structure », Transactions A.M.C.I., v. 114, 1949 .

(6) S. LexravsiY. - " Eplift in gravity dams 》, Conslable, London, 1958. 